\title{
Zum 75. Geburtstag von Michael Ermann
}

\author{
Cord Benecke • Anna Ursula Dreher · Jürgen Körner • \\ Ulrich Lamparter • Diana Pflichthofer • Timo Storck
}

Online publiziert: 7. November 2018

(C) Springer Medizin Verlag GmbH, ein Teil von Springer Nature 2018

Am 29. Oktober 2018 wurde Michael Ermann, der Gründer und federführende Herausgeber der Zeitschrift Forum der Psychoanalyse, 75 Jahre alt. Wir, die Herausgeber des Forums, gratulieren ihm sehr herzlich und danken ihm für sein unermüdliches Engagement für unsere Zeitschrift.

Die Idee, das Forum der Psychoanalyse zu gründen, stammte von ihm. Es war sein Anliegen, eine wissenschaftliche Zeitschrift ins Leben zu rufen, die einerseits theoretisch anspruchsvoll, andererseits aber auch klinisch fruchtbar sein sollte und dazu beitragen könnte, das in der Psychoanalyse oft unklare Verhältnis von Theorie und Praxis durchsichtiger zu machen. Außerdem schien ihm die Zeit gekommen zu sein, Brücken zu schlagen, über die historisch gezogenen Gräben zwischen den psychoanalytischen Fachgesellschaften in Deutschland. Er verwirklichte diese Ideen mit der Gründung von Forum der Psychoanalyse in der Zusammenarbeit mit Jürgen Körner und Sven Olaf Hoffmann und mit Unterstützung des Springer-Verlages im Jahre 1985.

Das Forum der Psychoanalyse erscheint nunmehr seit 33 Jahren, und wir können sehr zufrieden sein mit dem Erfolg unserer Zeitschrift. Die Idee, eine Plattform für lebendige, auch kontrovers geführte Diskussionen zur Verfügung zu stellen, wurde vielfach verwirklicht. Dazu hat Michael Ermann selbst maßgeblich beigetragen, nicht nur in seiner Funktion als federführender Herausgeber, sondern auch als $\mathrm{Au}-$ tor eigener Beiträge. Seine ihm eigene Offenheit für innovative Ideen und seine Toleranz gegenüber wissenschaftlich Andersdenkenden prägte auch das Forum der Psychoanalyse bis zum heutigen Tage.

Prof. Dr. J. Körner ( $\bowtie)$

Cimbernstr. 28, 14129 Berlin, Deutschland

E-Mail: juergen.koerner@ipu-berlin.de 
Wir wünschen Michael Ermann noch viele Jahre kreativen Schaffens, auch im Dienste unseres Forum der Psychoanalyse, für das wir mit ihm so gerne zusammenarbeiten.

Cord Benecke, Anna Ursula Dreher, Jürgen Körner, Ulrich Lamparter, Diana Pflichthofer und Timo Storck 